\title{
Thermal and electrical properties of polyimide/PANI nanofiber composites prepared via in situ polymerization
}

\author{
ASEEL A. KAREEM* \\ Department of Physics, College of Science, University of Baghdad, Iraq
}

\begin{abstract}
Polyimide/polyaniline nanofiber composites were prepared by in situ polymerization with various weight percentages of polyaniline (PANI) nanofibers. X-ray diffraction (XRD) and Fourier transform infrared spectra (FT-IR), proved the successful preparation of PANI nanofiber composite films. In addition, thermal stability of PI/PANI nanofiber composites was superior relative to PI, having $10 \%$ gravimetric loss in the range of $623{ }^{\circ} \mathrm{C}$ to $671{ }^{\circ} \mathrm{C}$ and glass transition temperature of $289{ }^{\circ} \mathrm{C}$ to $297{ }^{\circ} \mathrm{C}$. Furthermore, the values of the loss tangent $\tan \delta$ and $\mathrm{AC}$ conductivity $\sigma_{\mathrm{AC}}$ of the nanocomposite films were notably higher than those of pure polyimide. The addition of $5 \mathrm{wt} \%$ to $15 \mathrm{wt} . \%$ PANI nanofiber filler enhanced the activation energy of PI composites from $0.37 \mathrm{eV}$ to $0.34 \mathrm{eV}$.
\end{abstract}

Keywords: polyimide; polyaniline nanofiber; in situ polymerization; PI/PANI nanofiber composite films

\section{Introduction}

Polyimides (PI), as a kind of high-performance engineering polymers, have been widely used in many advanced technology fields due to their excellent thermal stability, outstanding mechanical properties and low dielectric constants [1]. In recent years, conducting polymers containing conjugating $\pi$-electron systems, such as polyaniline (PANI), polythiophene, and polypyrrole, have been widely studied. PANI has attracted special attention because of its easy polymerization, high yield at low cost, relatively high conductivities, redox reversibility, and environmental stability [2]. These properties provide its wide applications in batteries, molecular electronic devices, photoelectric cells, light emitting diodes, electromagnetic shielding and biosensors [3]. Moreover, the PANI of nanometer sizes $(<100 \mathrm{~nm})$ provides larger surface areas and smaller dimensions compared with their bulky counterparts, which permits enhanced interactions with the surrounding polymer matrices [4]. PANI nanofibers can be prepared by rapid mixing - a simple and fast method that presents the advantage of not using organic solvent.

*E-mail: aseelalobaedy@yahoo.com
However, applications of PANI have been limited due to its low solubility in common organic solvents and its difficult processability [5]. This drawback can be overcome by the dispersion and formation of a conductive network of PANI nanofibers within conventional polymers, following different approaches such as melt blending by extrusion, solution/dispersion, in situ polymerization, and electrochemical polymerization [6]. In this work, the electrical and thermal properties of prepared PI/PANI composite films by in situ polymerization have been studied. X-ray diffraction (XRD) and Fourier transform infrared spectra (FT-IR) were used to prove the successful preparation of PANI nanofiber composite films.

\section{Experimental}

The polyimide was prepared using two different monomers: pyromellitic dianhydride (PMDA) and p-phenylene diamine (PDA), commercially available from Sigma-Aldrich. $2 \mathrm{~g}$ (10 mmol) PDA and $40 \mathrm{~mL}$ NMP were placed in a flask and stirred for 30 min. $2 \mathrm{~g}$ (10 mmol) PMDA was then added to the solution in partition and stirred overnight at room temperature to prepare an approximately $20 \%$ poly(amic acid) (PAA) solution. 
$1 \mathrm{M} \mathrm{HCl}$ solution $(89.06 \mathrm{~mL})$ was prepared. The solution was divided into two parts labeled (a) and (b). The temperature of the solution was adjusted to $40{ }^{\circ} \mathrm{C}$ by use of a water bath. Aniline monomer $(7.67 \mathrm{~g})$ was dissolved in the part (a) of acidic solution and $14.67 \mathrm{~g}$ of ammonium persulfate (APS) oxidant was similarly dissolved in the part (b). The two solutions were mixed rapidly and the temperature was kept at $40^{\circ} \mathrm{C}$ for $3 \mathrm{~h}$.

In order to improve the removal of moisture and free dopant acid, the PANI nanofibers were washed repeatedly in a solution of water and acetone until $4 \mathrm{pH}$. Then, the sample was dried under vacuum in an oven at $80^{\circ} \mathrm{C}$ for $10 \mathrm{~h}$.

The nanocomposite films were prepared by thermal imidization of the PI/PANI nanofibers cast films. PANI nanofibers with different weight percentages $5 \mathrm{wt} . \%, 10 \mathrm{wt} . \%$ and $15 \mathrm{wt} . \%$ were added to the PAA solution and stirred at room temperature until a homogeneous solution was obtained; the resulting product was filtered, followed by storage in air at $80{ }^{\circ} \mathrm{C}$ for $1 \mathrm{~h}$. For thermal curing of the PI, the cast composite films were heat-treated at $200{ }^{\circ} \mathrm{C}$ for $3 \mathrm{~h}$ followed by $300{ }^{\circ} \mathrm{C}$ for $2 \mathrm{~h}$.

The thermal stability of the composites was evaluated by using a thermogravimetric analyzer (TGA) type: LINSEIS Simultaneous Thermal Analyzer (STA PT1000). The samples were heated using platinum crucibles from $0{ }^{\circ} \mathrm{C}$ to $900{ }^{\circ} \mathrm{C}$ at a heating rate of $10{ }^{\circ} \mathrm{C} / \mathrm{min}$ under nitrogen atmosphere. Dielectric parameters were measured by using LCR meter model (HP- 4275) at fixed frequency of $100 \mathrm{kHz}$.

\section{Results and discussion}

The prepared composite films were characterized by XRD. Fig. 1 shows the XRD patterns of the polyimide/PANI nanofiber composite films with various percentages of PANI nanofiber.

As is clearly seen in Fig. 1a, there is a peak at $2 \theta=22^{\circ}$ in the diffractogram of the polyimide and the non-Gaussian distribution pattern reveals a semi-crystalline structure polymer [7]. This peak has also been present in the all diffractograms of the polyimide composite films.
As the loading of PANI nanofibers increased in the PAA precursor (curves $b$ to $d$ ), the peaks shoulder heights at $2 \theta=22^{\circ}$ also increased, suggesting that this could be due to the formation of PANI nanofiber chain and increase of PANI nanofibers content in the polyimide matrix $[3,8]$.

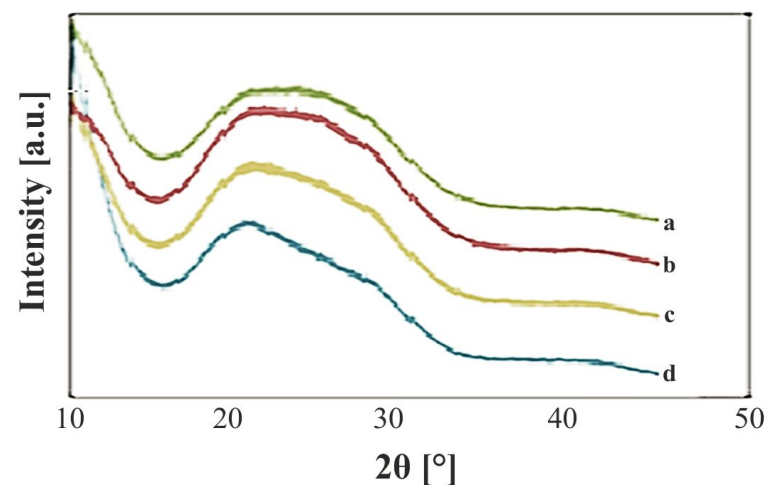

Fig. 1. XRD patterns of (a) pure polyimide and the polyimide composite films with (b) 5 wt. $\%$, (c) $10 \mathrm{wt} . \%$ and (d) $15 \mathrm{wt} . \%$ PANI nanofibers.

The FT-IR spectra of the prepared polyimide/PANI nanofiber composite films, with different percentages of PANI nanofiber, are depicted in Fig. 2.

The characteristic absorption bands of the imide groups near $1780 \mathrm{~cm}^{-1}, 1720 \mathrm{~cm}^{-1}$ and $1390 \mathrm{~cm}^{-1}$ were observed in the FT-IR spectra of the prepared samples after thermal imidization of the poly(amic acid)/PANI nanofiber precursor. Meanwhile, the characteristic absorption of the amide carbonyl at $1650 \mathrm{~cm}^{-1}$ did not appear in the spectra, indicating that the imidization reaction was complete [9].

The characteristic vibration bands of PANI at $1570 \mathrm{~cm}^{-1}$ and $1490 \mathrm{~cm}^{-1}$ can be associated with $\mathrm{C}=\mathrm{N}$ and $\mathrm{C}=\mathrm{C}$ stretching vibrations of quinone and benzene rings, respectively. As the content of PANI nanofibers increased, the intensity of $\mathrm{C}=\mathrm{C}$ band gradually became stronger in the FT-IR spectra of the polyimide/PANI nanofiber composite films [10].

Thermal stability of pure PI, PI/PANI nanofiber composite films with different wt.\% (5 wt.\%, 10 wt.\% and 15 wt.\%) of PANI nanofiber content was examined by TGA. Table 1 shows the initial 


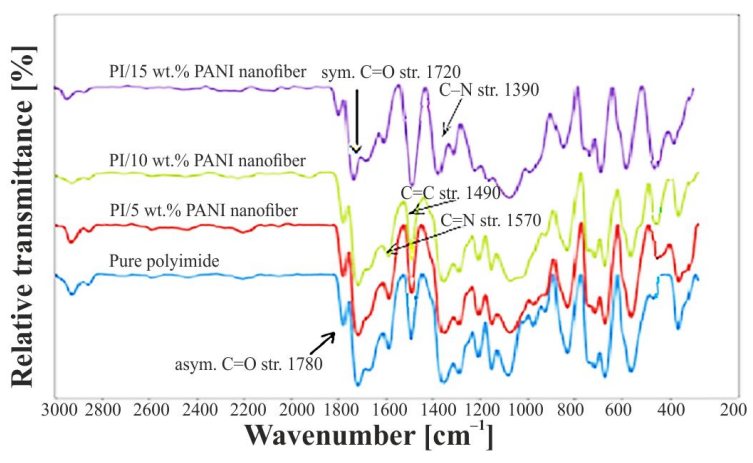

Fig. 2. Representative Fourier transform infrared (FT-IR) spectra of pure polyimide and its composite films with various percentages of PANI nanofibers.

decomposition temperature $\left(\mathrm{T}_{0}\right)$, at $10 \%$ weight loss $\left(\mathrm{T}_{10}\right)$, and maximum decomposition temperature $\left(\mathrm{T}_{\max }\right)$. As shown in Fig. 3, the major step of weight loss for PI occurs above $550{ }^{\circ} \mathrm{C}$. This behavior agrees with the literature [11]. The $\mathrm{T}_{0}$ occurs at $552{ }^{\circ} \mathrm{C}, \mathrm{T}_{10}$ around $581{ }^{\circ} \mathrm{C}$, and $\mathrm{T}_{\max }$ of $615^{\circ} \mathrm{C}$ has been observed $[11,12]$. In PI/PANI nanofiber composites, 5 wt.\% addition of PANI nanofibers into PI matrix resulted in improved thermal properties $\left(\mathrm{T}_{0}=573{ }^{\circ} \mathrm{C}, \mathrm{T}_{10}=623{ }^{\circ} \mathrm{C}\right.$, and $\left.\mathrm{T}_{\max }=678^{\circ} \mathrm{C}\right)$. PI/PANI nanofibers with $10 \mathrm{wt} . \%$ filler content in the polymer matrix have better thermal properties compared to PI with 5 wt.\% PANI nanofiber. Consequently, 15 wt.\% PI/PANI nanofibers showed further increase in stability, which would be beneficial for electronic devices [13, 14].

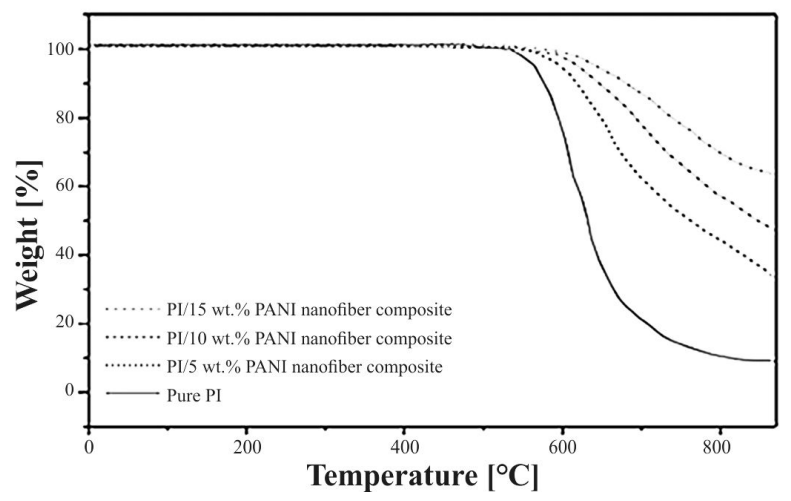

Fig. 3. TGA curves of pure PI and its composite films with various percentages of PANI nanofibers obtained at a heating rate of $10{ }^{\circ} \mathrm{C} / \mathrm{min}$ in $\mathrm{N}_{2}$.
Fig. 4 shows the loss tangent $(\tan \delta)$ of PI/PANI nanofiber composites with different percentages of PANI nanofibers, which attain the highest values at $\mathrm{Tg}$ of $289{ }^{\circ} \mathrm{C}$ and $297{ }^{\circ} \mathrm{C}$. The highest values of $\tan \delta$ and $\mathrm{Tg}$ for $15 \mathrm{wt} . \%$ PANI nanofiber suggest an increase in chain stiffness with filler loading. Furthermore, the values of $\tan \delta$ and $\mathrm{Tg}$ of the nanocomposite films are notably higher than for pure polyimide. In summary, the PANI nanofibers are promising candidates for fabricating high performance polyimide nanocomposites $[10,15]$.

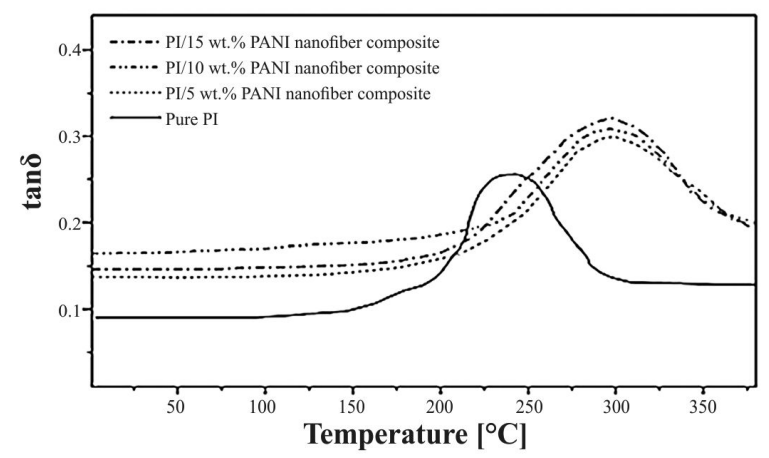

Fig. 4. Variation of loss tangent $(\tan \delta)$ with temperature for pure polyimide and its composite films with various percentages of PANI nanofibers.

Fig. 5 shows the dependence of AC conductivity on temperature at different percentages of PANI nanofibers. The $\sigma_{\mathrm{AC}}$ increases when temperature increases from $25{ }^{\circ} \mathrm{C}$ to $105{ }^{\circ} \mathrm{C}$. The increase in electrical conductivity of polyimide/PANI nanofiber composites may result from the increased chain ordering by annealing effect. Similar result has been reported reported by Al-Ajaj et al. [11].

The shape of the curves in Fig. 5 indicates that the relationship between $\ln \sigma$ and 1000/T is almost linear. This indicates that the AC conductivity of the composites follows Arrhenius equation:

$$
\sigma=\sigma_{0} \exp (E a / k T)
$$

where $\mathrm{k}$ is the Boltzmann constant, $\mathrm{T}$ is the temperature and $\mathrm{Ea}$ is the conduction activation energy which represents the minimum energy needed to overcome the potential barrier. 
Table 1. Thermal analysis data of pure PI and its composite films with various percentages of PANI nanofibers.

\begin{tabular}{lcccc}
\hline \multicolumn{1}{c}{ Polymer } & $\mathrm{T}_{\mathrm{g}}\left[{ }^{\circ} \mathrm{C}\right]$ & $\mathrm{T}_{0}\left[{ }^{\circ} \mathrm{C}\right]$ & $\mathrm{T}_{10}\left[{ }^{\circ} \mathrm{C}\right]$ & $\mathrm{T}_{\max }\left[{ }^{\circ} \mathrm{C}\right]$ \\
\hline \hline PI & 238 & 552 & 581 & 615 \\
PI/5 wt.\% PANI nanofiber composite & 289 & 573 & 623 & 678 \\
PI/10 wt.\% PANI nanofiber composite & 292 & 594 & 644 & 696 \\
PI/15 wt.\% PANI nanofiber composite & 297 & 598 & 671 & 778 \\
\hline
\end{tabular}

Table 2. Electrical activation energy values for pure polyimide and its composite films with various percentages of PANI nanofibers.

\begin{tabular}{lc}
\hline \multicolumn{1}{c}{ Sample } & Activation energy $[\mathrm{eV}]$ \\
\hline \hline Pure polyimide & 0.47 \\
PI/5 wt.\% PANI nanofiber composite & 0.37 \\
PI/10 wt.\% PANI nanofiber composite & 0.35 \\
PI/15 wt.\% PANI nanofiber composite & 0.34 \\
\hline
\end{tabular}

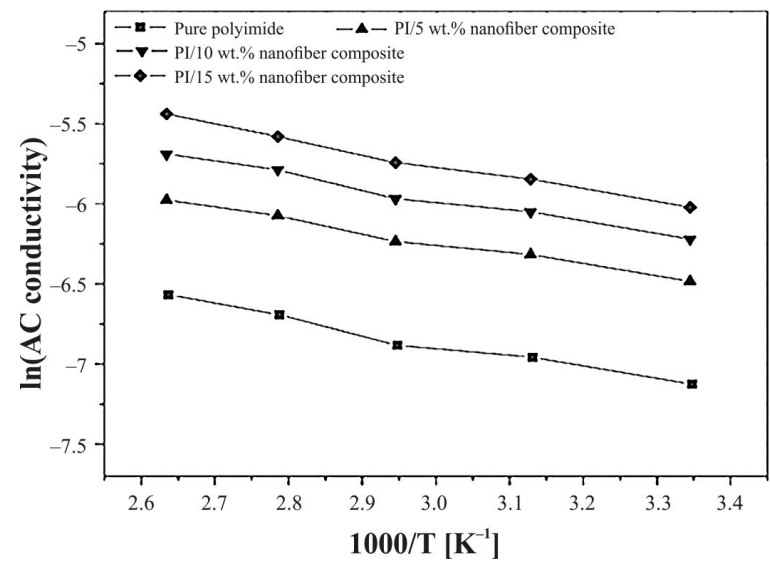

Fig. 5. Variation of $\ln$ of AC conductivity with 1000/T for pure polyimide and its composite films with various percentages of PANI nanofibers.

Table 2 includes the values of Ea obtained from the variation of $\sigma_{\mathrm{AC}}$ as a function of weight percentage of PANI nanofibers.

It can be seen that Ea values decrease from $0.37 \mathrm{eV}$ to $0.34 \mathrm{eV}$ as the weight percentages of PANI nanofibers increase, indicating that the energy gap of the nanocomposite becomes narrower due to creation of localized electrons states by heating, and thus electrons tunneling and hoping take place from the valence energy band to the conduction energy band $[13,16]$.
Polyaniline nanofibers based polyimide was found to improve the conductivity of corresponding nanocomposites. The improvement of conductivity in polyaniline nanofibers based polyimide resulted from inclusion of conducting polyaniline nanofibers into polyimide [16].

\section{Conclusions}

The structural, thermal and AC electrical properties of a set of nanocomposites from polyimide as a matrix and PANI nanofibers as a filler prepared by in situ polymerization were investigated as a function weight percentage of PANI nanofibers of $5 \mathrm{wt} . \%, 10 \mathrm{wt} . \%$ and $15 \mathrm{wt} . \%$. It was found that the successful preparation of polyimide/PANI nanofiber composites was confirmed by XRD and FT-IR studies. The PANI nanofiber addition increased the thermal stability of PI composites. The addition of $15 \%$ PANI nanofibers to the PI resulted in higher, by about $59^{\circ} \mathrm{C}$, Tg values of the composites. Higher $\tan \delta$ and Tg values of 15 wt.\% PANI nanofiber composite films suggest an increase in chain stiffness with filler loading. The values of $\tan \delta$ and $\sigma_{\mathrm{AC}}$ of the nanocomposite films were notably higher than those of pure polyimide. The addition PANI nanofibers as a filler enhanced the activation energy of PI composites. 


\section{References}

[1] WatcharaphalakoRn S., RUAngchuay L., Chotpattananont D., Sirivat A., Schwank J., Polym. Int., 54 (2005), 1126.

[2] Wang N., Chen Y., Ren J., Huang X., Chen X., Li G., LiU D., J. Polym. Res., 24 (2017), 42.

[3] Rezaei F., Tavandashti N.P., Zahedi A.R., Res. Chem. Intermed., 40 (3), 1233.

[4] Cho S., Kim M., LeE J.S., Jang J., ACS Appl. Mater. Inter., 7 (40) (2015), 22301.

[5] Ebrahim SH.M., Soliman M.M., Abd El-LatiF M.M., High. Perform. Polym., 22 (2010), 377.

[6] Zhou D., Subramaniam S., Mark J., J. Macromol. Sci. A, 42 (2005), 113.

[7] Ahmad M.B., Gharayebi Y., Salit M.S., HusSEIN M.Z., Ebrahimiasl S., DehZAngi A., Int. J. Mol. Sci., 13 (2012), 4860.

[8] Chen D., Miao Y., LiU T., ACS Appl. Mater. Inter., 5 (2013), 1206.
[9] Menshikova I.P., Pyshikina O.A., Levon K., Sergeyev V.G., Colloid J., 71 (2009), 233.

[10] Kausar A., Fiber Polym., 15 (2014), 2564.

[11] Al-Ajaj I.A., Kareem A.A., Mater. Sci.-Poland, 34 (2016), 132.

[12] Dai W., Yu J., Wang Y., Song Y., Bai H., Nishimura K., LiaO H., Jiang N., Macromol. Res., 22 (2014), 13233.

[13] Han M.G., Byun S.W., Im S.S., Polym. Adv. Technol., 13 (2002), 320.

[14] Li T., Hsu S.L., J. Phys. Chem. B, 114 (2010), 6825.

[15] Kausar A., J. Thermoplast. Compos. Mater., 29 (3) (2016), 312.

[16] Tommalieha M.J., Zihlifa A.M., Ragosta G., J. Exp. Nanosci., 6 (2011), 652.

Received 2017-09-08

Accepted 2018-03-15 